This is a pre-print of an article published in MRS Communications, 2018, Volume 8, Issue 3, pp. 995999. The final authenticated version is available online at: https://doi.org/10.1557/mrc.2018.124

\title{
Thermal and mechanical issues of high power laser diode Degradation
}

\author{
Jorge Souto, Jose Luis Pura, Juan Jimenez \\ GdS Optronlab, Ed.LUCIA \\ Paseo de Belen 19, Universidad de Valladolid, 47011 Valladolid, Spain
}

\begin{abstract}
A computational model for the evaluation of the thermomechanical effects that give rise to the catastrophic optical damage (COD) of laser diodes has been devised. The model traces the progressive deterioration of the device running in continuous wave $(\mathrm{cw})$ conditions. The local heating of the active layer locally leads to the onset of the plastic regime. As a result, dislocations and threads of dislocations grow across the active layers and lead to rapidly growing temperatures in the quantum well $(\mathrm{QW})$. The poor power dissipation under these conditions has been identified as the key factor driving the final degradation of the laser.
\end{abstract}

Figure for graphical abstract: Fig. 4 b

Corresponding author: Jorge Souto. E-mail: souto@eii.uva.es

\section{Introduction}

High power laser diodes under continuous wave (cw) operation are devices with extremely elevated internal power densities within their active regions. A very high percentage of that power is effectively converted into light, but over $25 \%$ is transformed into heat. Therefore, heat dissipation is a crucial point in the fabrication of reliable semiconductor lasers. Three main degradation processes have been identified for laser diodes: rapid, gradual and catastrophic failure modes [1]. Rapid degradation is 
This is a pre-print of an article published in MRS Communications, 2018, Volume 8, Issue 3, pp. 995999. The final authenticated version is available online at: https://doi.org/10.1557/mrc.2018.124

characterised by a sharp drop of the optical power output in a time scale of hours, during the initial operation of the device. This malfunction can be easily screened by burn-in tests. Gradual degradation is a slow process that involves an ongoing decrease of the laser output power when used in cw, and which eventually determines the lifetime of a device with normal operation life. Catastrophic failure, the mode on which we will focus in this work, is a sudden and almost instantaneous degradation of the device that can occur after a certain period of normal operation of the device. This failure mode is usually known as catastrophic optical damage (COD), it can take place either at the facet mirror or at interior zones of the laser cavity, and is associated with the generation of dense accumulations of defects.

The onset of COD has been experimentally connected to critical temperatures in the range $120-200{ }^{\circ} \mathrm{C}$, which must be reached so that the thermal runaway takes place [2-4]. The values of these experimental temperatures should be handled with care, as the size of the experimental probes with which these temperatures are measured are orders of magnitude larger than the active layers of the lasers, where the hot regions are locally generated [5]. Over the years, various physico-mathematical models have been proposed to explain the root causes of COD [6-9]. Our approach basically differs from those previous models on the relevant role that the mechanical degradation of the device plays for the onset of COD. The catastrophic degradation is described in terms of realistic temperature and stress distribution around a local heat source, and the factors leading to thermal runaway are discussed.

\section{Experimental}

In previous works $[5,10]$, we have developed a model that describes the catastrophic opticaldamage (COD) of GaAs/AlGaAs laser diodes as a sequence of events. The device ages gradually under continuous wave $(\mathrm{CW})$ operation as a result of the formation of point defects or small loops. Eventually, hot spots can be generated in the active region of the laser by non-radiative recombination either at surface states on the 
This is a pre-print of an article published in MRS Communications, 2018, Volume 8, Issue 3, pp. 995999. The final authenticated version is available online at: https://doi.org/10.1557/mrc.2018.124

mirror facets, or at tiny regions inside the cavity where point defects accumulate. Those hot spots trigger the COD.

The reduced thermal conductivity of the central layers of the devices was shown to play a fundamental role in the route to COD. The diodes under consideration are laser bars consisting of broad area AlGaAs/GaAs graded-index separate-confinement heterostructures (GRINSCH) mounted p-side down on the heat sink and emitting at 808 $\mathrm{nm}$, with output powers of up to a few tens of watts, which correspond to power densities that locally can reach $12 \mathrm{MW} / \mathrm{cm}^{2}$. A detailed description of the compositions and thicknesses of the layers, as well as a complete list of the physical parameters used for the calculations, are given in reference [10]. The same reference system is used for the present work.

We have previously assumed that the effective thermal conductivity of the AlGaAs active layers could be estimated after different theoretical models [11,12]. These models predict reduced cross-plane thermal conductivities that might be about two orders of magnitude lower than that of the bulk material. Nakwaski et al.[13] recently analysed those models and pointed out to some clearly non-physical results that could be derived from them, suggesting to rely on the available experimental results until a more satisfying model has been devised. In the present work, we have chosen to keep in line with this last approach. Therefore, we have worked with an initial ratio of $1 / 10$ for the effective cross-plane $\left(\mathrm{k}_{\mathrm{cp}}\right)$ to bulk ( $\mathrm{k}_{\text {bulk }}$ ) thermal conductivities for the $12 \mathrm{~nm} \mathrm{Al} \mathrm{Al}_{0.1} \mathrm{Ga}_{0.9} \mathrm{As} \mathrm{QW}$ [14-16], while we have allowed for in-plane $\left(\mathrm{k}_{\mathrm{ip}}\right)$ to cross-plane ratios ranging from 1 to 5 . That last value may be rather large considering some of the experimental data [16,17], though it has been theoretically expected to reach such a level for sufficiently thin layers [11]. Progressive reductions for the cross-plane thermal conductivity have also been considered, so that the outcome of the gradual degradation of this parameter could be assessed.

The thermomechanical evaluation of the device has been performed by means of finite element analysis (FEM). The calculations have been undertaken using the COMSOL MultiphysicsR suite. A full 3D model of the device was set up in order to provide a scheme by which the very local heating processes could be described. 
This is a pre-print of an article published in MRS Communications, 2018, Volume 8, Issue 3, pp. 995999. The final authenticated version is available online at: https://doi.org/10.1557/mrc.2018.124

\section{Results}

Figure 1 summarises the results for a numerical simulation conducted for a facet heat source of $6 \mathrm{MW} / \mathrm{cm}^{2}$, and for different values of $\mathrm{k}_{\mathrm{c}-\mathrm{p}}$ and $\mathrm{k}_{\mathrm{i}-\mathrm{p}}$. The heat source is fed by laser reabsorption. It absorbs a fraction of the laser power which increases as the degradation progresses. The defect region generating the heat source is spatially limited to the thickness of the QW (12 nm, z direction), while it extends up to 1 micrometre in the lateral direction (y). The plots show the variation of the temperature, as determined by the absorbed laser power and the thermal conductivity, both on the facet (subfigure a) of the device as well as inside the laser cavity (subfigure b). The first relevant information that can be derived from these graphs is that very high temperature gradients are generated both in the growth direction and towards the inner cavity along the QW. The extension of the hot spot speaks of a very local effect. In the vertical direction, the temperature outside the guide layers is almost identical to that of the heat sink (300 K); meanwhile, in the inner cavity, the temperatures are reduced to about $330 \mathrm{~K}$ for positions in the device just 125-150 nm apart from the facet, evidencing the very local character of the heat source.

The trends in terms of the thermal conductivity changes are similar for the facet and the interior of the laser. The gradual reduction of the in-plane to cross-plane conductivity ratio results in increasingly larger peak temperatures in the QW; the same can be argued as the cross-plane thermal conductivity diminishes. In both cases, the changes for the temperatures in the guide layers are much less significant than those observed in the QW. Therefore, for a fixed power absorption at the heat source, the reduction of the thermal conductivity results in increasing temperatures for the heated spot.

The sets of results from the thermal model were used as an input for the evaluation of the thermal stresses in the device [18]. The corresponding stress plots closely follow the temperature graphs in the central layers of the device, but an additional stress source is observed as the discontinuity in the compositional percentage of $\mathrm{Al}$ comes across the guide/QW interface ( $26 \%$ to $10 \%$ ). When a range of power values for the heat source was evaluated, the Tresca stress - peak temperature pairs were arranged in a similar way to what has been previously described $[5,10]$. All the data points are nearly aligned along a straight line in the Tresca stress vs peak temperature plot, as shown in Figure 2. 
This is a pre-print of an article published in MRS Communications, 2018, Volume 8, Issue 3, pp. 995999. The final authenticated version is available online at: https://doi.org/10.1557/mrc.2018.124

Experimental yield strength results for GaAs are also plotted in the graph. Within our COD model, the crossing of the yield strength plot and the straight line grouping the stress - temperature points marks the onset of plasticity leading to the final degradation of the device. As the plastic regime is reached, dislocations and clusters of dislocations are locally formed, and they trigger additional heating effects that will be described below. The temperature at which this final stage of degradation is reached may be somewhat in excess of the value read from the intersection of the straight line defined by the Tresca stress - temperature points and the yield strength curve. In fact, the mechanical strength is enhanced by the low dimensionality of the QW; therefore, one must consider that the intersection must occur at a higher temperature than it does for the bulk material. Therefore, assuming a Hall-Petch mechanism holds for the active layer, the onset of plasticity for the QW must be initiated at temperatures over $550 \mathrm{~K}$, well above the usually reported critical temperatures $[10,19]$.

The expected evolution of the degradation process is sketched in Figure 3. In the initial operational stages of the device, the temperature increases as a result of local power fluctuations whereas the thermal conductivity remains basically unaltered. Over an extended period of $\mathrm{cw}$ operation, the characteristics of the laser slowly deteriorate due to a progressive accumulation of point defects, as well as to other effects such as thermal lensing [20] or current-crowding [21]. All of these mechanisms result in locally enhanced power absorption levels. The critical temperature, associated with the onset of plasticity of the QW, is eventually reached, most likely at regions with high concentrations of nonradiative recombination centres, e.g. surface states or dense accumulations of defects inside the QW cavity. When this happens, dislocations will develop across the active layers of the device. This has a two-fold effect. On the one hand, the optical absorption drastically increases, as extrinsic absorption mechanisms are activated [22]. This results in an augmented absorption of the laser field in the vicinity of the degraded region, and the concomitant temperature rise. On the other hand, dislocations also affect the thermal conductivity. This effect is particularly relevant for systems with initially low concentrations of dislocations [23], as is the case for GaAs/AlGaAs based devices, for which those levels are below $10^{3}-10^{4} \mathrm{~cm}^{-2}$ for the state of the art fabrication processes. If that number is exceeded, as it would occur following the onset of the plastic deformation, recombination enhanced effects (REE) readily set in [24] and the number of dislocations rapidly grows. This may not have a severe effect on the cross-plane conductivity, which 
This is a pre-print of an article published in MRS Communications, 2018, Volume 8, Issue 3, pp. 995999. The final authenticated version is available online at: https://doi.org/10.1557/mrc.2018.124

is already drastically limited by the dimension of the QW and its surface roughness, but it will more acutely affect the in-plane thermal transport, as it will limit the path of the phonons in the corresponding directions. Therefore, the in-plane to cross-plane thermal conductivity ratio is expected to be notably reduced, while the cross-plane conductivity would initially diminish slightly. However, two-orders of magnitude reduction in thermal conductivity has been estimated for Si/Ge superlattices (SL) if dislocation densities of about $10^{12} \mathrm{~cm}^{-2}$ were present [25] and analogous reductions have been measured for GaN when the dislocation density scales from $\approx 10^{6} \mathrm{~cm}^{-2}$ to $10^{10} \mathrm{~cm}^{-2}[26]$, so that a severe drop of the cross-plane conductivity would also follow. The result of these drops in the thermal figures is, in any case, an increasing focalization of the heating effect. As the heat is less effectively spread out, the temperature in the hot spot of the laser grows in a correlated manner, and even more so if the increased absorption of the laser radiation is considered. The simultaneous occurrence of both effects sets up a perfect storm that, following a path analogous to the one outlined in Figure 3, eventually leads to the melting of the device.

Fig. 4 sums up under simple terms the evolution of the device under cw operation. In the early steps, the local heating of the QW generates thermal stresses in the central layers of the device which result in a thermal compression (a) that does not significantly alter the crystalline structure of the constitutive material. However, a gradual accumulation of point defects, responsible for the initially slow drop in the optical power delivered by the device, has been identified [27]. In the long run, local temperatures grow and the plastic onset is reached. When this happens (b), an irreversible mechanical deformation is caused, and threads of dislocations readily expand across the QW and the surrounding guiding layers.

\section{Discussion}

In our model, COD is triggered as the onset of plastic deformation is reached. If the mechanical degradation of the QW/guide layers is not considered, the increased laser light absorption that is often mentioned as the one of the main feedback effects that launch COD, does not seem to be sufficient to account for the eventual melting of the QW. According to our simulation, the extension of the heating effect inside the QW is limited 
This is a pre-print of an article published in MRS Communications, 2018, Volume 8, Issue 3, pp. 995999. The final authenticated version is available online at: https://doi.org/10.1557/mrc.2018.124

to about $125 \mathrm{~nm}$, as the rest of the cavity channel remains virtually transparent to the laser radiation.

Some preliminary calculations permit to assess the additional power absorption due to the laser attenuation within those $125 \mathrm{~nm}$. These have been conducted assuming that the laser is working under high power emission conditions $\left(12 \mathrm{MW} / \mathrm{cm}^{2}\right)$. The results detailed in Figure 1 permit to evaluate the temperature dependent absorption coefficient $(\alpha)$ of AlGaAs materials [28] as an explicit function of the position inside the QW. By substituting in the Beer-Lambert law, it could be determined that the power loss within that region would amount to less than $1 \%$ of the operational power for all the $\mathrm{k}_{\mathrm{cp}, \mathrm{p}} \geq 0.1$. $\mathrm{k}_{\text {bulk }}$ conditions considered in Figure 1. That is, in practice the net power absorption should be corrected in those cases from $6 \mathrm{MW} / \mathrm{cm}_{2}$ to, at most, $6.01 \mathrm{MW} / \mathrm{cm}^{2}$. However, if the effects of the mechanical degradation on the absorption coefficient are taken into account[22], those numbers are somewhat increased, and figures around 7\% would be reached. Even if it is a significant correction, the increased power absorption is still far from being relevant. Hence, the decrease of the thermal conductivity must play a key role in the degradation process. Note that, without considering any additional power dissipation in the heat source, the peak temperature rises from $525 \mathrm{~K}$ for $\mathrm{k}_{\mathrm{cp}}=0.1 \cdot \mathrm{k}_{\mathrm{bulk}}$; $\mathrm{k}_{\mathrm{ip}}=2.5 \cdot \mathrm{k}_{\mathrm{cp}}$, to $614 \mathrm{~K}$ for the isotropic $\mathrm{k}_{\mathrm{cp}}=0.1 \cdot \mathrm{k}_{\text {bulk }}$ case, and to 692 and $873 \mathrm{~K}$ for $\mathrm{k}_{\mathrm{cp}}=0.075 \cdot \mathrm{k}_{\text {bulk }}$ and $\mathrm{k}_{\mathrm{cp}}=0.05 \cdot \mathrm{k}_{\text {bulk }}$, respectively. If the conductivity decreased to the $\mathrm{k}_{\mathrm{cp}}=0.025 \cdot \mathrm{k}_{\text {bulk }}$ level, the QW would readily melt under the $6 \mathrm{MW} / \mathrm{cm}^{2}$ absorption condition.

The local temperatures would rise within the QW in a similar proportion as that just described for the maximum value, as seen in Figure 1. This point-by-point increase would also result in higher values for the absorption coefficient, so that, though the spatial extension of the heating effect does not significantly change, the power absorption would change accordingly to the enhanced temperature levels. A detailed evaluation of these modifications is under way, though the most relevant factor governing the activation of the COD seems to be the reduction of the thermal conductivity, without which the 
This is a pre-print of an article published in MRS Communications, 2018, Volume 8, Issue 3, pp. 995999. The final authenticated version is available online at: https://doi.org/10.1557/mrc.2018.124

feedback processes apparently would not reach the required levels to effectively bring about the final degradation of the device.

All in all, our model for COD differs from others that have been put forward to explain the drastic damage in diode lasers [29] fundamentally in the paramount role that the mechanical deterioration plays in the extrinsic feedback loop. If the diminished values of the thermal conductivities are not explicitly considered, our computations indicate that this feedback effect does not increase the local temperatures in such a way that thermal runaway is set-up; rather than that, a saturation regime would be reached, as already pointed out in refs [7,30]. The same runs for the intrinsic feedback loop: even more so, given that the absorption coefficient increases as the concentration of dislocations grows. These considerations are summarised in Figure 5, where both feedback loops (intrinsic and extrinsic) are represented. This figure emphasizes the physical roots behind the scheme: the generation of thermal stresses, the eventual reaching of the plastic regime, and the massive generation of dislocations that bring forward the relevant changes in the thermal and optical properties of the active layers.

\section{Conflict of interest disclosure}

The authors have no competing financial interests.

\section{Acknowledgments}

This work has been funded by the Spanish Government (ENE2014-56069-C4-4-R) and Junta de Castilla y Leon (VA29U13 and VA081U16). J.L. Pura wishes to acknowledge a grant by the FPU programme of the Spanish Government (FPU14/00916).

\section{References}

[1] O. Ueda: On degradation studies of III-V compound semiconductor optical devices over three decades: Focusing on gradual degradation. Jpn. J. Appl. Phys. 49, 090001 (2010). doi:10.1143/JJAP.49.090001.

[2] M. A. Bettiati: High optical strength GaAs-based laser structures. Microelectron. 
This is a pre-print of an article published in MRS Communications, 2018, Volume 8, Issue 3, pp. 995999. The final authenticated version is available online at: https://doi.org/10.1557/mrc.2018.124

Reliab. 53, 1496-1500 (2013). doi:10.1016/j.microrel.2013.07.121.

[3] W.C. Tang, H.J. Rosen, P. Vettiger and D.J. Webb: Raman microprobe study of the time development of AlGaAs single quantum well laser facet temperature on route to catastrophic breakdown. Appl. Phys. Lett. 58, 557-559 (1991). doi:10.1063/1.104585.

[4] J.W. Tomm, M. Ziegler, M. Oudart, J. Nagle and J. Jimenez: Gradual degradation of GaAs-based quantum well lasers, creation of defects, and generation of compressive strain. Phys. Status Solidi Appl. Mater. Sci. 206, 1912-1915 (2009). doi:10.1002/pssa.200881455.

[5] J. Souto, J.L. Pura and J. Jimenez: About the physical meaning of the critical temperature for catastrophic optical damage in high power quantum well laser diodes.

Laser Phys. Lett. 13, 025005 (2016). doi:10.1088/1612-2011/13/2/025005.

[6] W. Nakwaski: Thermal analysis of the catastrophic mirror damage in laser diodes. $J$.

Appl. Phys. 57, 2424-2430 (1985). doi:10.1063/1.334350.

[7] G. Chen and C.L. Tien: Facet heating of quantum well lasers. J. Appl. Phys. 74, 2167- 2174 (1993). doi:10.1063/1.354750.

[8] U. Menzel: Self-consistent calculation of facet heating in asymmetrically coated edge emitting diode lasers. Semicond. Sci. Technol. 13, 265-276 (1998). doi:10.1088/02681242/13/3/004.

[9] W.R. Smith: Mathematical modelling of thermal runaway in semiconductor laser operation. 8276, (2003). doi:10.1063/1.373538.

[10] J. Souto, J.L. Pura and J. Jimenez: Nanoscale effects on the thermal and mechanical properties of $\mathrm{AlGaAs} / \mathrm{GaAs}$ quantum well laser diodes: influence on the catastrophic 
This is a pre-print of an article published in MRS Communications, 2018, Volume 8, Issue 3, pp. 995999. The final authenticated version is available online at: https://doi.org/10.1557/mrc.2018.124

optical damage (COD). J. Phys. D. Appl. Phys. 50, 235101 (2017). doi:10.1088/13616463/aa6fbd.

[11] G. Chen and C.L. Tien: Thermal conductivities of quantum well structures. $J$.

Thermophys. Heat Transf. 7, 311-318 (1993). doi:10.2514/3.421.

[12] L.H. Liang and B. Li: Size-dependent thermal conductivity of nanoscale semiconducting systems. Phys. Rev. B - Condens. Matter Mater. Phys. 73, 1-4 (2006).

doi:10.1103/PhysRevB.73.153303.

[13] E. Gęsikowska and W. Nakwaski: An impact of multi-layered structures of modern optoelectronic devices on their thermal properties. Opt. Quantum Electron. 40, 205216 (2008). doi:10.1007/s11082-007-9151-z.

[14] W. Capinski, H. Maris, T. Ruf, M. Cardona, K. Ploog and D. Katzer: Thermal conductivity measurements of GaAs/AlAs superlattices using a picosecond optical pumpand-probe technique. Phys. Rev. B. 59, 8105-8113 (1999). doi:10.1103/PhysRevB.59.8105.

[15] H. Tan, K.K. Kamath, Z. Mi, P. Bhattacharya and D. Klotzkin: Analysis of the reduced thermal conductivity in InGaAs/GaAs quantum dot lasers from chirp characteristics. Appl. Phys. Lett. 89, 1-3 (2006). doi:10.1063/1.2354415.

[16] M.N. Luckyanova, J.A. Johnson, A.A. Maznev, J. Garg, A. Jandl, M.T. Bulsara, E.A. Fitzgerald, K.A. Nelson and G. Chen: Anisotropy of the thermal conductivity in GaAs / AlAs superlattices Anisotropy of the thermal conductivity in GaAs / AlAs superlattices.

Nano Lett. 13, 3973-3977 (2013). doi:10.1021/n14001162.

[17] J. Piprek, J. Kolodzey and C.S. Ih: Thermal Conductivity Reduction in GaAs - AlAs Distributed Bragg Reflectors. Technology. 10, 81-83 (1998). 
This is a pre-print of an article published in MRS Communications, 2018, Volume 8, Issue 3, pp. 995999. The final authenticated version is available online at: https://doi.org/10.1557/mrc.2018.124

[18] A. Martin-Martin, M. Avella, M.P. Iniguez, J. Jimenez, M. Oudart and J. Nagle: Thermomechanical model for the plastic deformation in high power laser diodes during operation. J. Appl. Phys. 106, 073105 (2009). doi:10.1063/1.3236507.

[19] N.J. Petch: The cleavage strength of polycrystals. J. Iron Steel Inst. 174, 25-28 (1953).

[20] Y. Sin, N. Presser, M. Brodie, Z. Lingley, B. Foran and S.C. Moss: Degradation mechanisms in high-power multi-mode InGaAs-AlGaAs strained quantum well lasers for high-reliability applications. Proc. SPIE. 9348, 93480L-1-93480L-12 (2015). doi:10.1117/12.2076788.

[21] P.G. Eliseev, A.G. Glebov and M. Osiński: Current self-distribution effect in diode lasers: Analytic criterion and numerical study. IEEE J. Sel. Top. Quantum Electron. 3, 499-506 (1997). doi:10.1109/2944.605700.

[22] S. Adachi: Optical properties of GaAs partially amorphized by ion implantation: Effective- medium-approximation analysis. J. Appl. Phys. 69, 7768-7773 (1991). doi:10.1063/1.347504.

[23] P.E. Hopkins and J.C. Duda: Introduction to Nanoscale Thermal Conduction. Heat Transf. - Math. Model. Numer. Methods, Inf. Technol. 112, 872-881 (2006). doi:10.5772/13590.

[24] L. Sugiura: Comparison of degradation caused by dislocation motion in compound semiconductor light-emitting devices. Appl. Phys. Lett. 70, 1317-1319 (1997). doi:10.1063/1.118523.

[25] G. Chen, M. Neagu and T. Borca-Tasciuc: Thermal Conductivity and Heat Transfer in Superlattices. MRS Proc. 478, 12-15 (1997). doi:10.1557/PROC-478-85.

[26] C. Mion, J.F. Muth, E. a. Preble and D. Hanser: Accurate dependence of gallium nitride thermal conductivity on dislocation density. Appl. Phys. Lett. 89, 092123 (2006). doi:10.1063/1.2335972. 
This is a pre-print of an article published in MRS Communications, 2018, Volume 8, Issue 3, pp. 995999. The final authenticated version is available online at: https://doi.org/10.1557/mrc.2018.124

[27] Y. Sin, N. Presser, Z. Lingley, M. Brodie, B. Foran and S.C. Moss: Reliability, failure modes, and degradation mechanisms in high power single- and multi-mode InGaAsAlGaAs strained quantum well lasers. Proc. SPIE. 9733, 973304-1-97330413 (2016). doi:10.1117/12.2208802.

[28] B. Monemar, K.K. Shih and G.D. Pettit: Some optical properties of the AlxGa1-xAs alloys system. J. Appl. Phys. 47, 2604-2613 (1976). doi:10.1063/1.322979.

[29] J.W. Tomm, M. Ziegler, M. Hempel and T. Elsaesser: Mechanisms and fast kinetics of the catastrophic optical damage (COD) in GaAs-based diode lasers. Laser Photon. Rev.

5, 422-441 (2011). doi:10.1002/lpor.201000023.

[30] R. Schatz and C.G. Bethea: Steady state model for facet heating leading to thermal runaway in semiconductor lasers. J. Appl. Phys. 76, 2509-2521 (1994). doi:10.1063/1.358509.

\section{Figure Captions}

Figure. 1. Computed temperatures for a facet heat source with power density of 6 $\mathrm{MW} / \mathrm{cm}^{2}$, and for the ranges of cross-plane and in-plane effective thermal conductivities specified in the figure: (a) front facet mirror temperature profile across the growth direction as a function of the distance from the centre of the QW, and (b) profile inside the laser cavity as a function of the distance from the front facet mirror.

Figure 2. Experimental yield strength of bulk GaAs, and estimated maximum Tresca stress - peak temperature pairs for the same ranges of effective thermal conductivities specified in the previous figure, and for heat power densities ranging from 2 to 10 $\mathrm{MW} / \mathrm{cm}_{2}$ with a $2 \mathrm{MW} / \mathrm{cm}^{2}$ step size between consecutive points.

Figure 3. Symbols: computed peak temperatures in the device for heat power densities ranging from 2 to $12 \mathrm{MW} / \mathrm{cm}^{2}$ with a $2 \mathrm{MW} / \mathrm{cm}^{2}$ step size, displaying the progressive 
This is a pre-print of an article published in MRS Communications, 2018, Volume 8, Issue 3, pp. 995999. The final authenticated version is available online at: https://doi.org/10.1557/mrc.2018.124

increase of the temperature as the in-plane and cross-plane thermal conductivities degrade. Line: prospective degradation path illustrating the gradual shift from the curve with the initial thermal conductivity parameters towards an abrupt temperature increase when the thermal conductivity is drastically reduced by the generation of dislocations.

Figure 4. Scheme illustrating the degradation of the device: (a) heat gradients develop across the central layers of the laser structure and give rise to mechanical stresses; (b) once the onset of plasticity is reached, massive generation of dislocations take place, propagating and rapidly multiplying across the QW and the guides.

Figure 5. Proposed mechanisms driving the thermal and mechanical deterioration of the laser diode that eventually drive the COD. As illustrated, and according to our estimations, the intrinsic loop would just lead to a saturation of the heating effect, so that the mechanical degradation of the device must be invoked in order to effectively trigger the extrinsic loop that leads to thermal runaway. 
This is a pre-print of an article published in MRS Communications, 2018, Volume 8, Issue 3, pp. 995999. The final authenticated version is available online at: https://doi.org/10.1557/mrc.2018.124
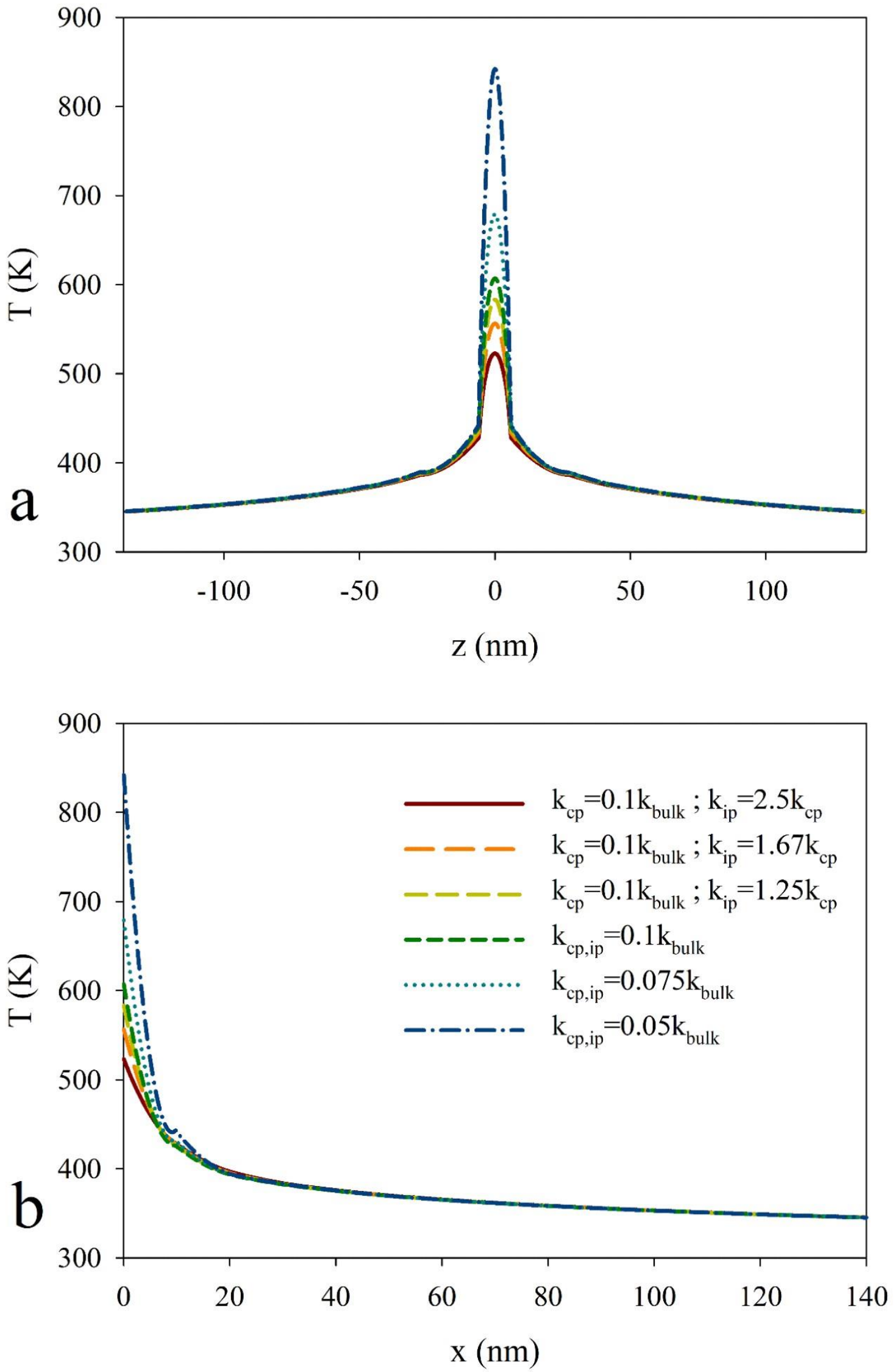

Figure 1 
This is a pre-print of an article published in MRS Communications, 2018, Volume 8, Issue 3, pp. 995999. The final authenticated version is available online at: https://doi.org/10.1557/mrc.2018.124
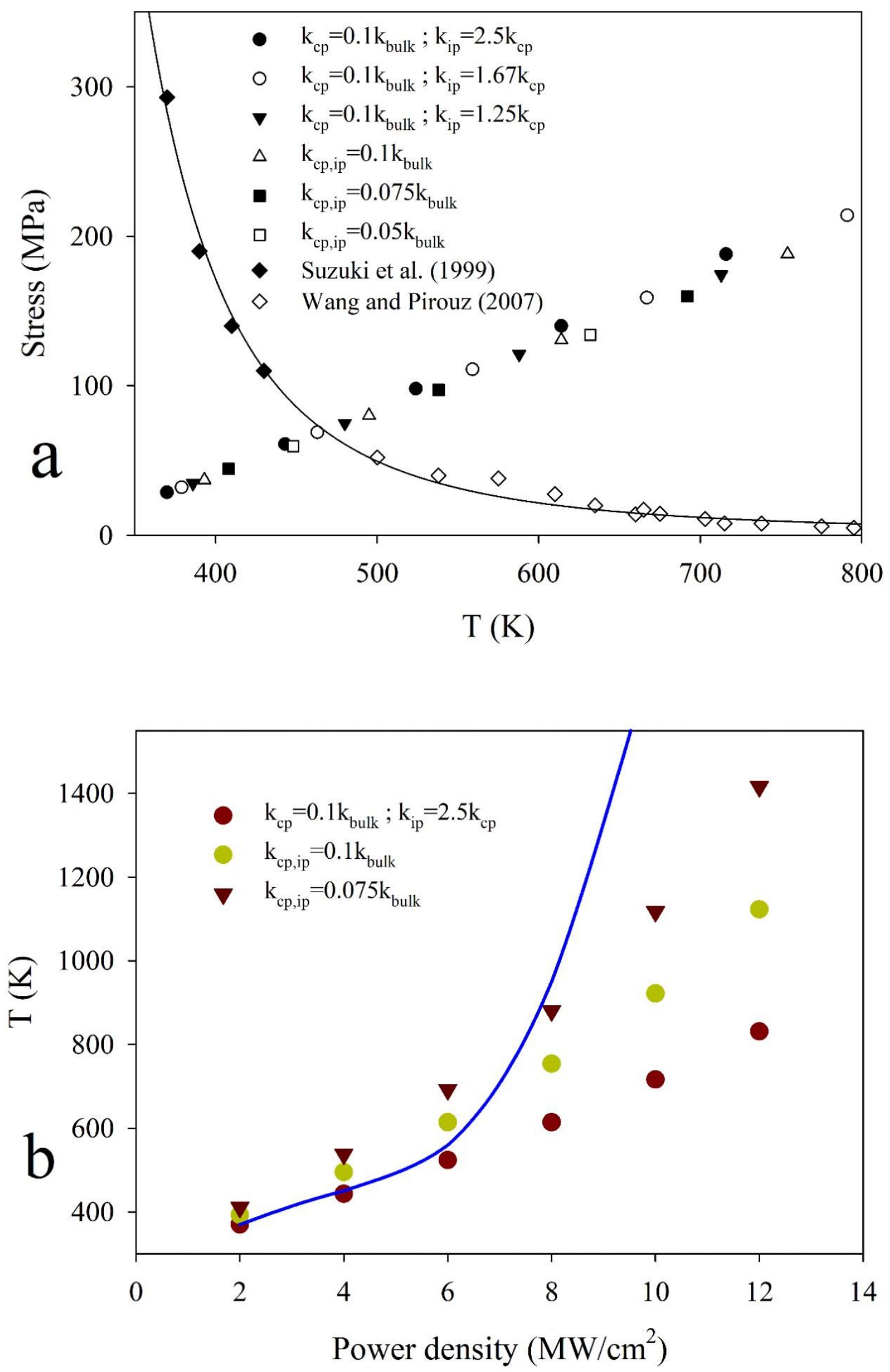

Figure 2 
This is a pre-print of an article published in MRS Communications, 2018, Volume 8, Issue 3, pp. 995999. The final authenticated version is available online at: https://doi.org/10.1557/mrc.2018.124

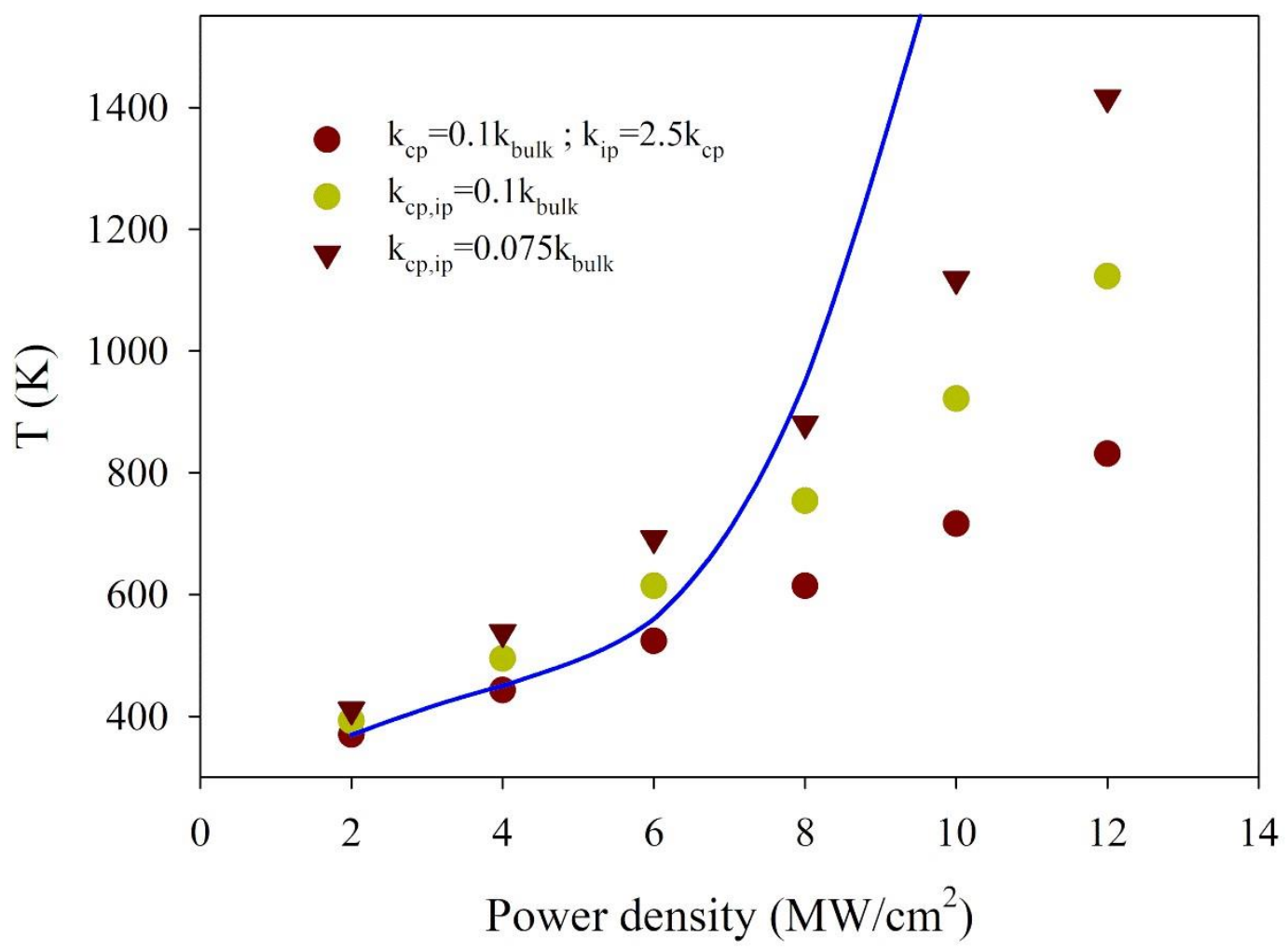

Figure 3 
This is a pre-print of an article published in MRS Communications, 2018, Volume 8, Issue 3, pp. 995999. The final authenticated version is available online at: https://doi.org/10.1557/mrc.2018.124

\section{Heat source}

\section{QW thermally compressed}

a

\section{Onset of plasticity}

b

Figure 4

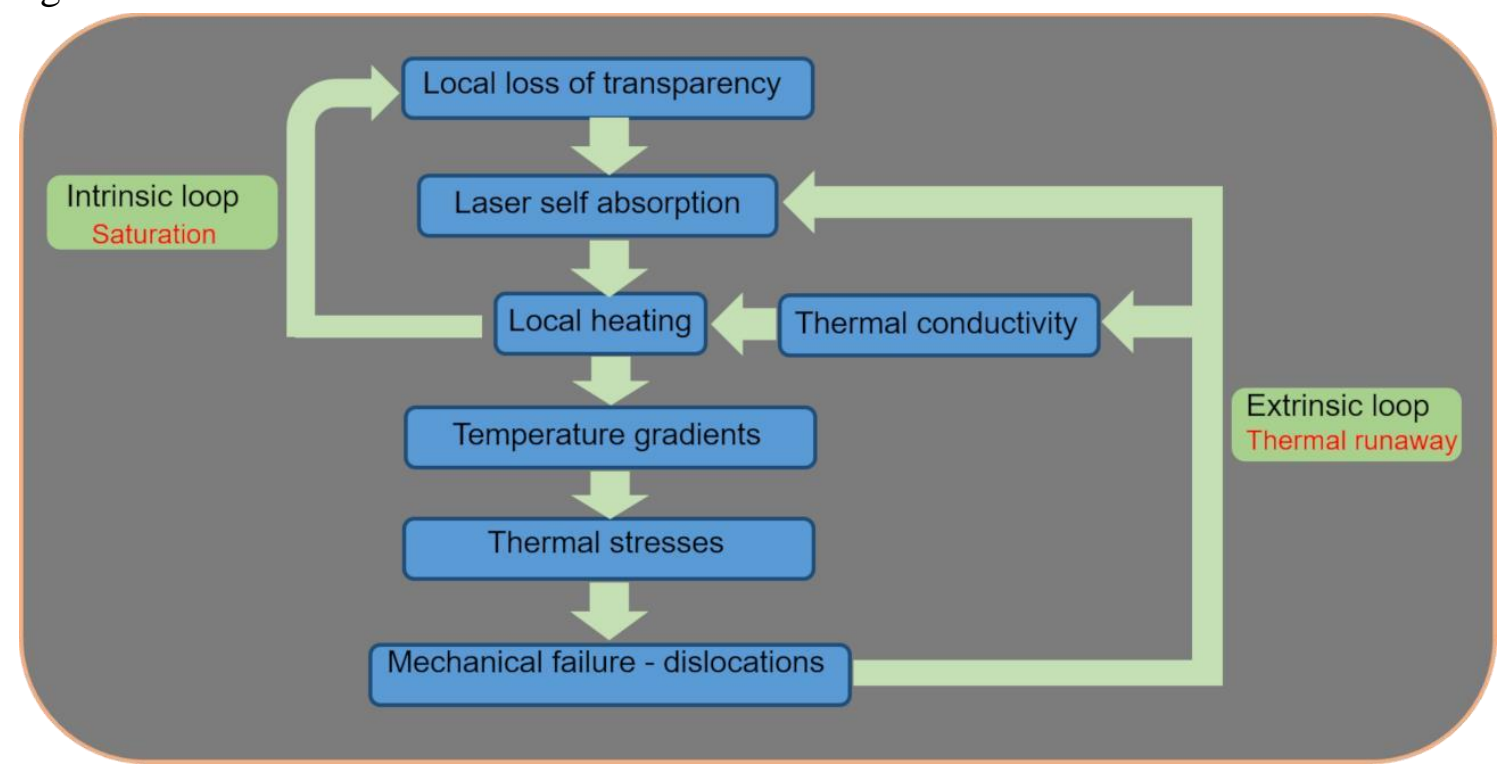


This is a pre-print of an article published in MRS Communications, 2018, Volume 8, Issue 3, pp. 995999. The final authenticated version is available online at: https://doi.org/10.1557/mrc.2018.124

Figure 5 